\title{
CMR feature tracking strain patterns and their association with circulating cardiac biomarkers in patients with hypertrophic cardiomyopathy
}

\author{
Ersin Cavus $^{1}$ (D) $\cdot$ Kai Muellerleile ${ }^{1} \cdot$ Samuel Schellert $^{1} \cdot$ Jan Schneider $^{1} \cdot$ Enver Tahir $^{2} \cdot$ Celeste Chevalier $^{1}$. \\ Charlotte Jahnke ${ }^{1}$ - Ulf K. Radunski ${ }^{3} \cdot$ Gerhard Adam $^{2} \cdot$ Paulus Kirchhof $^{1} \cdot$ Stefan Blankenberg ${ }^{1} \cdot$ Gunnar K. Lund $^{2}$. \\ Maxim Avanesov ${ }^{4} \cdot$ Monica Patten ${ }^{1}$
}

Received: 27 December 2020 / Accepted: 17 March 2021 / Published online: 29 March 2021

(c) The Author(s) 2021

\begin{abstract}
Aims CMR feature tracking strain (CMR-FT) provides prognostic information. However, there is a paucity of data in hypertrophic cardiomyopathy (HCM). We sought to analyze global CMR-FT parameters in all four cardiac chambers and to assess associations with NT-proBNP and cardiac troponin T (hsTnT) in patients with HCM.

Methods This retrospective study included $144 \mathrm{HCM}$ patients and 16 healthy controls with CMR at $1.5 \mathrm{~T}$. Analyses were performed on standard steady-state free precession cine (SSFP) CMR data using a commercially available software. Global

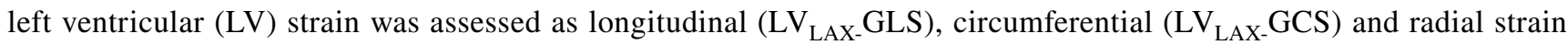
$\left(\mathrm{LV}_{\mathrm{LAX}}\right.$ GRS) on long -axis (LAX) and as $\mathrm{LV}_{\mathrm{SAX}} \mathrm{GCS}$ and $\mathrm{LV}_{\mathrm{SAX}}$-GRS on short- axis (SAX). Right ventricular (RV-GLS), left atrial (LA-GLS) and right atrial (RA-GLS) strain were assessed on LAX.

Results We found $\mathrm{LV}_{\mathrm{LAX}}{ }^{-\mathrm{GLS}}[-18.9(-22.0,-16.0),-23.5(-25.5,-22.0) \%, p=0.0001), \mathrm{LV}_{\mathrm{SAX}} \mathrm{GRS}^{\mathrm{G}}$ [86.8 (65.9115.5), 119.6 (91.3-143.7) \%, $p=0.001$ ] and $\mathrm{LA}_{\mathrm{LAX}}{ }^{-\mathrm{GLS}}$ [LA $\mathrm{LCH}^{-\mathrm{GLS}} 29.2$ (19.1-37.7), $\mathrm{LA}_{2 \mathrm{CH}^{-}}{ }^{-\mathrm{GLS}} 38.2(34.3-47.1) \%$, $p=0.0036 ; \mathrm{LA}_{4 \mathrm{CH}^{-}} \mathrm{GLS} 22.4$ (14.6-30.7) vs. $\mathrm{LA}_{4 \mathrm{CH}^{-}} \mathrm{GLS} 33.4$ (28.4-37.3) \%, $p=0.0033$ ] to be impaired in HCM compared to healthy controls despite normal LVEF. Furthermore, LV and LA strain parameters were impaired in HCM with elevated NT-proBNP and/or hsTnT, despite preserved LVEF compared to HCM with normal biomarker levels. There was a moderate correlation of LV and LA CMR-FT with levels of NT-proBNP and hsTnT.

Conclusion CMR-FT reveals LV and LA dysfunction in HCM despite normal LVEF. The association between impaired LV strain and elevated NT-proBNP and hsTnT indicates a link between unapparent functional abnormalities and disease severity in HCM.
\end{abstract}

Maxim Avanesov and Monica Patten authors contributed equally.

Ersin Cavus

ersin.cavus@uke.de

1 Clinic of Cardiology, University Heart and Vascular Center Hamburg Eppendorf, Martinistr.52, 20246 Hamburg,

Germany

2 Department of Diagnostic and Interventional Radiology, University Hospital Hamburg Eppendorf, Hamburg, Germany

3 Department of Cardiology and Angiology, Regio Clinics, Elmshorn and Pinneberg, Germany

4 Department of Radiology, AK Segeberger Kliniken GmbH, Bad Segeberg, Germany 


\section{Graphic abstract}

Typical CMR-FT findings in patients with hypertrophic cardiomyopathy

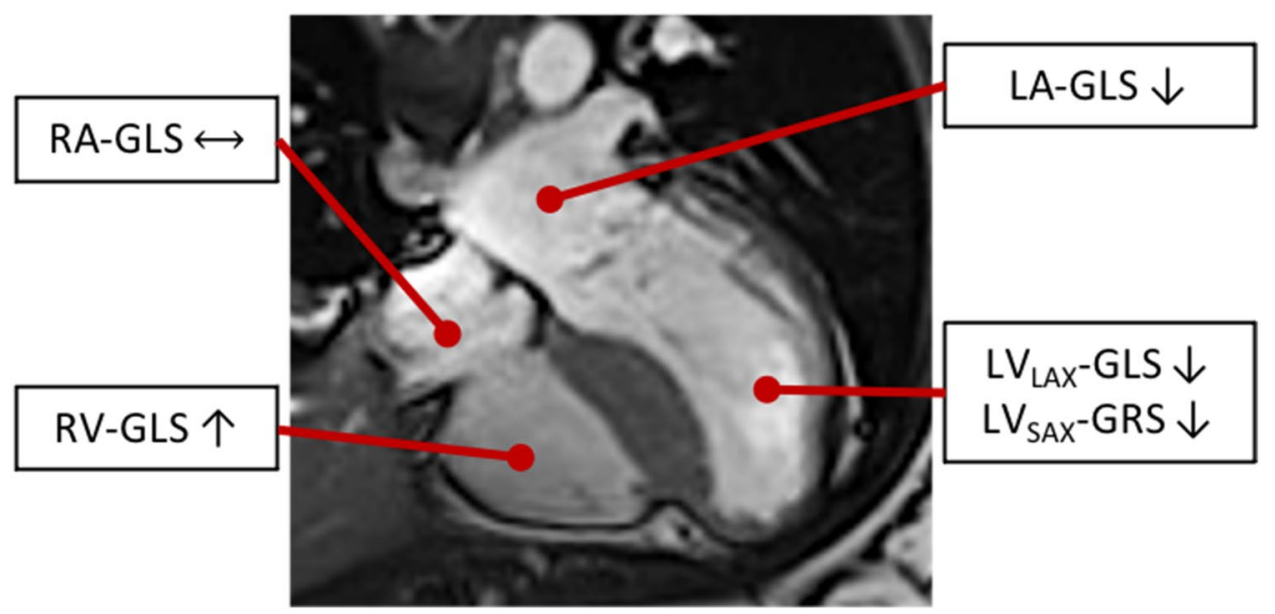

Keywords Strain imaging $\cdot$ CMR feature tracking strain $\cdot$ Hypertrophic cardiomyopathy $\cdot$ Cardiac biomarker

\begin{tabular}{|c|c|}
\hline \multicolumn{2}{|c|}{ Abbreviations } \\
\hline CMR-FT & $\begin{array}{l}\text { Cardiac magnetic resonance feature track- } \\
\text { ing strain }\end{array}$ \\
\hline $\mathrm{HCM}$ & Hypertrophic cardiomyopathy \\
\hline $\mathrm{HF}$ & Heart failure \\
\hline hsTnT & High-sensitivity troponin $\mathrm{T}$ \\
\hline $\mathrm{LA}_{2 \mathrm{CH}^{-}}-\mathrm{GLS}$ & $\begin{array}{l}\text { Left atrium two-chamber global longitudi- } \\
\text { nal strain }\end{array}$ \\
\hline $\mathrm{LA}_{4 \mathrm{CH}^{-}}-\mathrm{GLS}$ & $\begin{array}{l}\text { Left atrium four-chamber global longitudi- } \\
\text { nal strain }\end{array}$ \\
\hline LGE & Late gadolinium enhancement \\
\hline LVEF & Left ventricular ejection fraction \\
\hline $\mathrm{LV}_{\mathrm{LAX}} \mathrm{GCS}$ & $\begin{array}{l}\text { Left ventricular long-axis global circumfer- } \\
\text { ential strain }\end{array}$ \\
\hline $\mathrm{LV}_{\mathrm{LAX}} \mathrm{GLS}$ & $\begin{array}{l}\text { Left ventricular long-axis global longitudi- } \\
\text { nal strain }\end{array}$ \\
\hline LV $_{\text {LAX }}$ GRS & $\begin{array}{l}\text { Left ventricular long-axis global radial } \\
\text { strain }\end{array}$ \\
\hline $\mathrm{LV}_{\mathrm{SAX}} \mathrm{GCS}$ & $\begin{array}{l}\text { Left ventricular short-axis global circumfer- } \\
\text { ential strain }\end{array}$ \\
\hline $\mathrm{LV}_{\mathrm{SAX}} \mathrm{GRS}$ & $\begin{array}{l}\text { Left ventricular short-axis global radial } \\
\text { strain }\end{array}$ \\
\hline NT-proBNP & $\begin{array}{l}N \text {-terminal prohormone of the brain natriu- } \\
\text { retic peptide }\end{array}$ \\
\hline PSIR & Phase-sensitive inversion recovery \\
\hline RA-GLS & Right atrium global longitudinal strain \\
\hline RV-GLS & Right ventricle global longitudinal strain \\
\hline SCD & Sudden cardiac death \\
\hline SSFP & Steady-state free precession \\
\hline STI & Speckle tracking imaging \\
\hline
\end{tabular}

\section{Background}

Hypertrophic cardiomyopathy (HCM) is characterized by inappropriate and mostly asymmetric left ventricular (LV) hypertrophy, resulting from disarrays of fibers and fascicles, dysmorphic myocytes and accumulation of myocardial fibrosis [1]. It represents the most frequent cause of sudden cardiac death in young people, thus early diagnosis and initiation of therapy can prevent life-threatening events [2]. Nevertheless, assessing myocardial function in HCM by cardiac imaging is challenging since conventional parameters, i.e. left ventricular ejection fraction (LVEF), are often preserved or supernormal in HCM patients despite clinical heart failure symptoms [2]. In the past, echocardiographybased strain analyses (speckle tracking imaging (STI)) were able to detect regional and global myocardial dysfunction in patients with HCM with a superior prognostic value for major adverse cardiac events compared to LVEF assessment [3]. In particular, impaired global longitudinal strain $\left(\mathrm{LV}_{\mathrm{LAX}} \mathrm{GLS}\right)$, was associated with major cardiovascular events in HCM [4]. CMR feature tracking strain (CMR-FT) has the ability to assess early changes in myocardial mechanics more sensitive compared to echocardiography due to the excellent image quality across the complete left and right ventricle of steady-state free precession (SSFP) cine CMR [5]. CMR-FT has been used to assess LV strain in several studies in recent years, indicating incremental prognostic information in different cardiac diseases such as dilatative cardiomyopathy, ischemic cardiomyopathy, and HCM [6]. However, most available study populations were small and a comprehensive approach in HCM including all cardiac 
chambers is missing. The aim of our retrospective study was to analyze global CMR-FT strain parameters in all four cardiac chambers of HCM patients. Furthermore, we aimed to assess potential associations with well-established cardiac biomarkers such as NT-proBNP and troponin T (hsTnT).

\section{Methods}

\section{Patients and controls}

This retrospective study included 144 consecutive patients with HCM and 16 healthy subjects as controls. HCM patients were outpatients, in stable health condition and underwent clinically indicated CMR between Jun 2006 and Nov 2017. HCM was defined by a wall thickness $\geq 15 \mathrm{~mm}$ in one or more LV myocardial segments according to current guidelines of the ESC [2]. Exclusion criteria included coronary artery disease, relevant valvular dysfunction, post-operative status (myectomy, alcohol septal ablation), glomerular filtration rate $($ GFR $)<30 \mathrm{ml} / \mathrm{min} / 1.73 \mathrm{~m}^{2}$. The control group consisted of recently published healthy individuals [7]. Diastolic dysfunction (DD) was assessed and categorized by echocardiography in agreement with current guidelines [8]. Blood samples of all participants were obtained routinely \pm 3 days before/after CMR. Troponin $\mathrm{T}$ was measured by a high-sensitive assay and an established cutoff was applied with $\geq 14 \mathrm{pg} / \mathrm{mL}$ using the 99th percentile for defining abnormal values [9]. The NT-proBNP cutoff was set at $125 \mathrm{pg} / \mathrm{mL}$ according to the current guidelines [10]. The study followed the principles outlined in the Declaration of Helsinki and was approved by the local ethics committee. All patients and controls gave their written informed consent to use CMR information for research purposes.

\section{CMR protocol}

Clinically indicated CMR was performed on a 1.5-T scanner (Achieva, Philips Medical Systems, Best, the Netherlands). For the assessment of LV volumes and function, standard retrospectively gated SSFP cine CMR was performed in short- and in long-axis [two-chamber $(2 \mathrm{CH})$, three-chamber $(3 \mathrm{CH})$, four-chamber view $(4 \mathrm{CH})]$. Typical imaging parameters were: voxel size $1.36 \times 1.36 \times 6 \mathrm{~mm}^{3}$, echo time $=1.67 \mathrm{~ms}$, time to repetition $=3.34 \mathrm{~ms}$, flip angle $=60^{\circ}$, parallel acquisition technique $=$ SENSE. Late gadolinium enhancement $($ LGE) images were acquired using a phase-sensitive inversion recovery (PSIR) sequence at least ten minutes after bolus injection of contrast media, $0.075 \mathrm{mmol} / \mathrm{kg}$ Gd-BOPTA (MultiHance ${ }^{\circledR}$ ). Imaging parameters were as follows: voxel size $1.36 \times 1.36 \times 8 \mathrm{~mm}^{3}$, echo time $=2.40 \mathrm{~ms}$, time to repetition $=5.50 \mathrm{~ms}$, flip angle $=15^{\circ}$. Since $\mathrm{T} 1 \mathrm{mapping}$ was routinely introduced not until 2011 in our institution, we did not include T1 mapping in this analysis.

\section{CMR data analysis}

CMR data analysis was performed by two trained observers who were blinded to all clinical information. Commercially available post-processing software (Medis Suite MR, QMass ver. 8.1.74.2, QStrain ver. 2.0.70.2, Leiden, The Netherlands) was used to assess volumes and function of all cardiac chambers. LV and RV volumes, as well as LV mass and LV maximal wall thickness, were obtained from cine SAX. LA and RA volumetry were obtained from cine LAX as recommended [11].

CMR-FT measurements were performed using the QStrain application of Medis Suite MR [12]. To generate the most accurate tracing points, endo- and epicardial contours were manually traced in QMass and then copied to QStrain. In agreement with current recommendations LV strain was based on endo- and epicardial contours in shortand long axis, whereas RV, LA and RA contours were tracked from endocardium in LAX [5], [13-16]. Global CMR-FT was assessed as longitudinal (GLS), radial (GRS) and circumferential strain (GCS) in LV; in LA, RA and RV GLS were assessed. Negative values represent shortening of myocardium and positive values represent thickening referred to the direction of the deformation process (longitudinal, radial, circumferential). LA/RA maximum was defined at end-systole and LA/RA minimum at end-diastole. LGE was assessed semi-quantitatively as recommended for clinical indications [11] on PSIR images according to the 17-segment model of the AHA [17].

\section{Statistical analysis}

Statistical analysis was performed using MedCalc for Windows, version 12.7.7.0 (MedCalc Software, Ostend, Belgium). Continuous data are presented as median and IQR. Categorical data are presented as numbers and percentage. Inter-observer agreement was assessed by intra-class correlation coefficients (ICC) and ICC values indicated poor $(<0.5)$, moderate $(0.5-0.75)$, good $(0.75-0.9)$ or excellent $(>0.9)$ reliability [18]. Continuous data were compared using the Mann-Whitney test. Categorical data were analyzed using the chi-quadrat test or Fisher exact test, where appropriate. Correlations between continuous parameters were analyzed by spearman's correlation coefficient (rho). Statistical significance was set to $p<0.05$ without correction for multiple testing. 


\section{Results}

\section{Inter-observer agreement CMR-FT strain}

Inter-observer agreement of CMR-FT strain parameters was as follows: ICC $\operatorname{LV}_{\mathrm{LAX}}-\mathrm{GLS} 0.95(0.93,0.96), \mathrm{LV}_{\mathrm{LAX}}-\mathrm{GCS}$ $0.84(0.78,0.89)$, LV $_{\text {LAX }}-$ GRS $0.40(0.17,0.57)$, LV $_{\text {SAX }}-$ GRS $0.89(0.85,0.92), \mathrm{LV}_{\mathrm{SAX}}{ }^{-} \mathrm{GCS} 0.66(0.53,0.76), \mathrm{LA}_{2 \mathrm{CH}^{-}} \mathrm{GLS}$ 0.97 (0.96, 0.98), LA LCH$^{-}$GLS 0.97 (0.95, 0.98), RV-GLS $0.94(0.92,0.96)$, and RA-GLS $0.94(0.91,0.95)$.

\section{HCM patients and controls}

LV Mass index (LVMi), maximal LV wall thickness (LVWT) and median LA volumes were significantly higher in HCM patients compared to controls (Table 1). Eightyseven $(91.6 \%)$ of 95 HCM patients with sufficient echocardiography had diastolic dysfunction $\geq$ II (Table 2). Median $\mathrm{LV}_{\mathrm{LAX}}-\mathrm{GLS}$ of HCM patients was significantly lower compared to controls $[-18.9(-22.0,-16.0),-23.5(-25.5$, $-22.0) \%, p=0.0001$; Table 1, Fig. 1). Furthermore, median $\mathrm{LV}_{\mathrm{SAX}}-\mathrm{GRS}, \mathrm{LA}_{2 \mathrm{CH}^{-}}-\mathrm{GLS}$ and $\mathrm{LA}_{4 \mathrm{CH}^{-}}-\mathrm{GLS}$ were significantly lower in the HCM group compared to controls (Table 1, Fig. 1). In contrast, median RV-GLS was significantly higher in HCM compared to controls [- $38.0(-43.6,-33.9)$, $-31.4(-34.3,-28.3) \%, p=0.0002$; Table 1$)$.

\section{NT-proBNP}

104 (84\%) HCM patients had elevated NT-proBNP levels. There were no significant differences in major clinical and conventional CMR characteristics between HCM patients with and without elevated NT-proBNP levels (Table 2). HCM patients with elevated NT-proBNP had a significantly higher extent of LGE. $\mathrm{LV}_{\mathrm{LAX}}-\mathrm{GLS}, \mathrm{LV}_{\mathrm{LAX}}-\mathrm{GCS}$, $\mathrm{LV}_{\mathrm{LAX}}{ }^{-\mathrm{GRS}}, \mathrm{LV}_{\mathrm{SAX}}-\mathrm{GCS}, \mathrm{LV}_{\mathrm{SAX}}-\mathrm{GRS}$ and global longitudinal LA strain were all significantly lower in HCM patients with elevated NT-proBNP (Table 2, Fig. 1). There were no significant differences in RA and RV strain between both groups (Table 2). There were significant correlations of NT-proBNP levels with $\mathrm{LV}_{\mathrm{LAX}}-\mathrm{GLS}$ and $\mathrm{LV}_{\mathrm{SAX}} \mathrm{x}^{-\mathrm{GRS}}$ $\left(\mathrm{LV}_{\mathrm{LAX}}-\mathrm{GLS}: \quad r=0.492, \quad p<0.0001 ; \mathrm{LV}_{\mathrm{SAX}} \mathrm{GRS}^{-\mathrm{G}}\right.$ $r=-0.300, p=0.0007$, Fig. 2).

\section{Troponin T}

$44 \mathrm{HCM}$ patients had elevated hsTnT levels $(\geq 14 \mathrm{pg} / \mathrm{mL})$ (Table 3). There were no significant differences in major clinical characteristics, but a significantly lower (but normal) median LVEF as well as higher LV/LA volumes and higher LVMi in HCM patients with elevated hsTnT levels
(Table 3). Diastolic dysfunction $\geq$ stage II was present in the majority of both groups but less frequent in the group with hsTnT $\geq 14 \mathrm{pg} / \mathrm{mL}$ ( 81.3 vs. $96.5 \%, p=0.0233$ ). The presence of LGE was similar in both groups, but HCM patients with elevated hsTnT had a significantly higher extent of LGE (Table 3). $\mathrm{LV}_{\mathrm{LAX}}-\mathrm{GLS}, \mathrm{LV}_{\mathrm{LAX}}-\mathrm{GCS}$, $\mathrm{LV}_{\mathrm{LAX}}-\mathrm{GRS}, \mathrm{LV}_{\mathrm{SAX}}-\mathrm{GCS}, \mathrm{LV}_{\mathrm{SAX}}-\mathrm{GRS}$ and global longitudinal LA strain were all significantly lower in the group of HCM patients with elevated hsTnT (Table 3). $\mathrm{LV}_{\mathrm{LAX}}{ }^{-\mathrm{GLS}}(r=0.463, p<0.0001)$ and $\mathrm{LV}_{\mathrm{SAX}}{ }^{-\mathrm{GRS}}$ correlated significantly with hsTnT levels $(r=-0.321$, $p=0.0004$; Fig. 2).

\section{Discussion}

This study analyzed global myocardial strain patterns in all four cardiac chambers in patients with HCM by CMR-FT strain. The major findings were the following: first, we found $\mathrm{LV}_{\mathrm{LAX}}-\mathrm{GLS}, \mathrm{LV}_{\mathrm{SAX}}-\mathrm{GRS}$ and $\mathrm{LA}_{\mathrm{LAX}}-\mathrm{GLS}$ to be impaired in HCM patients compared to healthy controls despite normal LVEF in both groups (Table 1, Fig. 1). Second, all global LV and LA strain parameters were impaired in HCM patients with elevated NT-proBNP and hsTnT levels, despite preserved LVEF and a similar degree of diastolic dysfunction (Tables 2, 3). Third, we found significant but modest correlations of LV and LA CMR-FT strain with NT-proBNP and hsTnT levels (Fig. 2).

\section{LV and RV strain in HCM}

We found significantly decreased median $\mathrm{LV}_{\mathrm{LAX}}-\mathrm{GLS}$ and $\mathrm{LV}_{\text {SAX }}$-GRS despite preserved LVEF in HCM patients compared to healthy controls. Although LVEF is by far the most important clinical measure of LV function [19], it tends to overestimate LV systolic function in hypertrophied ventricles [2]. Therefore, more advanced approaches to assess myocardial function in HCM are desirable. CMR-FT offers many different advantages; it traces the cardiac deformation process precisely and reflects local forces (Fig. 3: arrows show direction and proportion of motion), displays disproportional LV thickening (note the reduced amplitude of voxel motion in CMR-FT strain in Fig. $4 \mathrm{a}-\mathrm{h}$ ), is well validated and has been used in a variety of cardiovascular diseases [20]. Moreover, $\mathrm{LV}_{\mathrm{LAX}}-\mathrm{GLS}$ and $\mathrm{LV}_{\mathrm{SAX}}-\mathrm{GRS}$ provide incremental prognostic information to LVEF in ischemic and non-ischemic cardiac disease $[3,21]$ and are associated with an increased risk of mortality and poor cardiovascular outcome in HCM [6]. We suspect that the association of strain with myocardial fibrosis [22] and scaring [23] could explain this observation.

In contrast, we found RV-GLS to be higher in HCM compared to controls (Table 1). RV function was neglected in 
Table 1 Clinical characteristics

\begin{tabular}{|c|c|c|c|}
\hline Parameter, unit & Controls $(n=16)$ & $\operatorname{HCM}(n=144)$ & $p$ value \\
\hline Age, years & $51(46-58)$ & $55(43-64)$ & 0.2191 \\
\hline Male, $n,(\%)$ & $8(50)$ & $88(61)$ & 0.4277 \\
\hline Heart rate, bpm & $61(57-76)$ & $65(59-73)$ & 0.6327 \\
\hline Height, m & $1.73(1.63-1.76)$ & $1.74(1.66-1.82)$ & 0.5636 \\
\hline Weight, kg & $78(70-88)$ & $82(71-90)$ & 0.4110 \\
\hline Creatinine, $\mathrm{mg} / \mathrm{dL}$ & $0.79(0.66-0.90)$ & $0.9(0.80-1.10)$ & 0.0220 \\
\hline GFR, $\mathrm{mL} / \mathrm{min}$ & $97(91-109)$ & $78(70-88)$ & 0.0037 \\
\hline Creatine kinase, U/L & $127(65-184)$ & $115(79.5-171)$ & 0.8737 \\
\hline hsTroponin T, pg/mL & $3(3-4)$ & $12(7-21.5)^{\mathrm{a}}$ & $<0.0001$ \\
\hline NT-proBNP, pg/mL & $46(32-97)$ & $581(227-1542)^{\mathrm{b}}$ & $<0.0001$ \\
\hline \multicolumn{4}{|l|}{ Volumes and functions } \\
\hline LVEF, $\%$ & $65(59-68)$ & $64(58-71)$ & 1.0000 \\
\hline LVEDVi, $\mathrm{mL} / \mathrm{m}^{2}$ & $85(79-90)$ & $88(78-99)$ & 0.6842 \\
\hline LVESVi, $\mathrm{mL} / \mathrm{m}^{2}$ & $29(26-38)$ & $30(24-40)$ & 0.9410 \\
\hline $\mathrm{LV}$ mass index, $\mathrm{g} / \mathrm{m}^{2}$ & $45(36-58)$ & $76(57-95)$ & $<0.0001$ \\
\hline Maximal LVWT, mm & $8.9(8.2-9.9)$ & $17.9(15.6-21.4)$ & $<0.0001$ \\
\hline RVEF, $\%$ & $62(57-65)$ & $64(58-68)$ & 0.5153 \\
\hline $\mathrm{RVEDVi}, \mathrm{mL} / \mathrm{m}^{2}$ & $80(67-89)$ & $73(64-88)$ & 0.4712 \\
\hline $\mathrm{RVESVi}, \mathrm{mL} / \mathrm{m}^{2}$ & $29(23-35)$ & $27(21-35)$ & 0.5832 \\
\hline LAEDVi, $\mathrm{mL} / \mathrm{m}^{2}$ & $14(12-17)$ & $36(22-56)$ & $<0.0001$ \\
\hline LAESVi, mL/m² & $39(31-41)$ & $54(41-73)$ & 0.0002 \\
\hline RAEDVi, mL/m² & $22(16-25)$ & $22(16-32)$ & 0.4050 \\
\hline $\mathrm{RAESVi}, \mathrm{mL} / \mathrm{m}^{2}$ & $40(28-43)$ & $38(27-48)$ & 1.0000 \\
\hline LGE presence, $n,(\%)$ & $0 / 16(0)$ & $104 / 143(73)$ & $<0.0001$ \\
\hline $\begin{array}{l}\text { LGE extent, } n \text { (amount of } \\
\text { LGE segments in } \% \text { ) }\end{array}$ & $0 / 272(0)$ & $308 / 2448(12.6)$ & $<0.0001$ \\
\hline Atrial fibrillation, $n,(\%)$ & 0 & $7(4.9)$ & $<0.0001$ \\
\hline HCM-SCD-score, $\%$ & 0 & $3.3(2.2-5.5)$ & $<0.0001$ \\
\hline \multicolumn{4}{|l|}{ Strain parameters, $\%$} \\
\hline $\mathrm{LV}_{\mathrm{LAX}}-\mathrm{GLS}$ & $-23.5(-25.5,-22.0)$ & $-18.9(-22.0,-16.0)$ & 0.0001 \\
\hline $\mathrm{LV}_{\mathrm{LAX}}{ }^{-\mathrm{GCS}}$ & $-25.0(-26.9,-23.2)$ & $-22.9(-26.4,-20.0)$ & 0.0848 \\
\hline $\mathrm{LV}_{\text {LAX }}-\mathrm{GRS}$ & $96.4(75.4-114.1)$ & 87.5 (68.9-107.4) & 0.3113 \\
\hline $\mathrm{LV}_{\mathrm{SAX}}-\mathrm{GCS}$ & $-23.3(-27.9,-21.1)$ & $22.1(-24.8,-18.3)$ & 0.1696 \\
\hline $\mathrm{LV}_{\mathrm{SAX}}-\mathrm{GRS}$ & $119.6(91.3-143.7)$ & $86.8(65.9-115.5)$ & 0.0014 \\
\hline $\mathrm{LA}_{2 \mathrm{CH}^{-}}-\mathrm{GLS}$ & $38.2(34.3-47.1)$ & $29.2(19.1-37.7)$ & 0.0036 \\
\hline $\mathrm{LA}_{4 \mathrm{CH}^{-}}-\mathrm{GLS}$ & $33.4(28.4-37.3)$ & $22.4(14.6-30.7)$ & 0.0033 \\
\hline RV-GLS & $-31.4(-34.3,-28.3)$ & $-38.0(-43.6,-33.9)$ & 0.0002 \\
\hline RA-GLS & $29.8(24.1-35.1)$ & $31.1(23.5-39.2)$ & 0.6959 \\
\hline
\end{tabular}

Statistical significance was defined as $p<0.05$. Significant results are highlighted in bold

Values are median [first (Q1) and third (Q3) quartiles] for continuous and $n$ (\% of total column number) for categorical data. HCM indicates hypertrophic cardiomyopathy, $b p m$, beats per minute, $h s$ high-sensitive, $N T N$-terminal, $L V$ left ventricular, $R V$ right ventricular, $L A$ left atrial, $R A$ right atrial, $E F$ ejection fraction, $E D V i$ end-diastolic volume index, $E S V i$ end-systolic volume index, $L V W T$ left ventricular wall thickness, $L G E$ late gadolinium enhancement, $S C D$-Score sudden cardiac death score, $L A X$ longitudinal axis, $S A X$ short axis, GLS global longitudinal strain, GCS global circumferential strain, GRS global radial strain, $2 C H$ two-chamber view, $4 \mathrm{CH}$ four-chamber view

${ }^{a}$ hsTnT was available in $n=119$

${ }^{\mathrm{b}} \mathrm{NT}$-proBNP was available in $n=124$

most cardiac diseases for a long time, but in recent years, the awareness is increasing $[24,25]$. Hypothetically, the "supra-normal" RV-GLS we found could indicate a compensatory reaction to increased LV filling pressure and requires 
Table 2 Clinical and CMR characteristics of HCM patients with and without elevated NT-proBNP

\begin{tabular}{|c|c|c|c|}
\hline Parameter, unit & $\begin{array}{l}\text { NT-proBNP } \\
<125 \mathrm{pg} / \mathrm{mL} \\
(n=20)\end{array}$ & $\begin{array}{l}\text { NT-proBNP } \\
\geq 125 \mathrm{pg} / \mathrm{mL} \\
(n=104)\end{array}$ & $p$ value \\
\hline Age, years & $49(38-57)$ & $57(45-65)$ & 0.0631 \\
\hline Male, $n,(\%)$ & $18(90)$ & $60(57.7)$ & 0.0054 \\
\hline Heart rate, bpm & $65(57-72)$ & $66(59-73)$ & 0.4713 \\
\hline Height, m & $1.79(1.72-1.84)$ & $1.73(1.65-1.82)$ & 0.0616 \\
\hline Weight, kg & $88(81-92)$ & $82(73-90)$ & 0.1065 \\
\hline Creatinine, $\mathrm{mg} / \mathrm{dL}$ & $1.0(0.9-1.2)$ & $0.9(0.8-1.1)$ & 0.0268 \\
\hline GFR, $\mathrm{mL} / \mathrm{min}$ & $79(66-97)$ & $80(68-100)$ & 0.5543 \\
\hline Creatine kinase, U/L & $138(118-170)$ & $108(73-169)$ & 0.1108 \\
\hline hsTroponin $\mathrm{T}, \mathrm{pg} / \mathrm{mL}$ & $5(3-8)$ & $13(8-27)$ & $<0.0001$ \\
\hline \multicolumn{4}{|l|}{ Volumes and functions } \\
\hline LVEF, \% & $67(61-72)$ & $64(57-69)$ & 0.0569 \\
\hline LVEDVi, $\mathrm{mL} / \mathrm{m}^{2}$ & $86(77-104)$ & 89 (79-99) & 0.9729 \\
\hline LVESVi, $\mathrm{mL} / \mathrm{m}^{2}$ & $28(21-39)$ & $33(27-42)$ & 0.1237 \\
\hline LV mass index, $\mathrm{g} / \mathrm{m}^{2}$ & $71(57-86)$ & $79(60-98)$ & 0.0796 \\
\hline Maximal LVWT, mm & $16.3(14.7-17.7)$ & $18.6(15.8-21.4)$ & 0.0182 \\
\hline RVEF, \% & $63(58-67)$ & $64(57-68)$ & 0.9864 \\
\hline RVEDVi, $\mathrm{mL} / \mathrm{m}^{2}$ & $85(68-102)$ & $73(63-88)$ & 0.0267 \\
\hline $\mathrm{RVESVi}, \mathrm{mL} / \mathrm{m}^{2}$ & $32(26-39)$ & $27(22-36)$ & 0.1006 \\
\hline $\mathrm{LAEDVi}, \mathrm{mL} / \mathrm{m}^{2}$ & $23(17-26)$ & $38(26-58)$ & 0.0001 \\
\hline LAESVi, $\mathrm{mL} / \mathrm{m}^{2}$ & $49(42-59)$ & $57(43-79)$ & 0.1747 \\
\hline RAEDVi, $\mathrm{mL} / \mathrm{m}^{2}$ & $23(17-27)$ & $22(16-34)$ & 0.6828 \\
\hline $\mathrm{RAESVi}, \mathrm{mL} / \mathrm{m}^{2}$ & $42(36-48)$ & $36(27-49)$ & 0.1707 \\
\hline Normal diastolic Function, $n,(\%)$ & $0 / 12(0)$ & $1 / 83(1.2)$ & 1.0000 \\
\hline $\mathrm{DD} \mathrm{I}, n,(\%)$ & $0 / 12(0)$ & $7 / 83(8.4)$ & 0.5901 \\
\hline $\mathrm{DD} \mathrm{II}^{\circ}, n,(\%)$ & 11/12(92) & $61 / 83(73.5)$ & 0.2821 \\
\hline $\mathrm{DD} \mathrm{III}^{\circ}, n,(\%)$ & $1 / 12(8)$ & $14 / 83$ & 0.6837 \\
\hline $\mathrm{DD} \geq \mathrm{II}^{\circ}, n,(\%)$ & $12 / 12(100)$ & $75 / 83(90.3)$ & 0.5901 \\
\hline NYHA I, $n,(\%)$ & $7 / 20(35)$ & $32 / 99(32.3)$ & 0.7996 \\
\hline NYHA II, $n,(\%)$ & $11 / 20(55)$ & $45 / 99(45.5)$ & 0.4703 \\
\hline NYHA III, $n,(\%)$ & $2 / 20(10)$ & $22 / 99(22.2)$ & 0.3587 \\
\hline NYHA IV, $n,(\%)$ & $0 / 20(0)$ & 0/99 (0) & 1.0000 \\
\hline LGE presence, $n,(\%)$ & $11 / 20(55)$ & 78/104 (75) & 0.1013 \\
\hline $\begin{array}{l}\text { LGE extent, } n \text { (amount of LGE segments } \\
\text { in \%) }\end{array}$ & $\begin{array}{l}19 / 340 \\
(5.6)\end{array}$ & $\begin{array}{l}233 / 1768 \\
(13.2)\end{array}$ & $<0.0001$ \\
\hline Atrial fibrillation, $n,(\%)$ & $0(0)$ & $4(3.8)$ & 1.0000 \\
\hline HCM-SCD-Score, \% & $2.6(1.8-6.1)$ & $3.5(2.2-5.0)$ & 0.3939 \\
\hline LVOT obstruction, $n,(\%)$ & $4(20)$ & $39(37.5)$ & 0.1990 \\
\hline \multicolumn{4}{|l|}{ Strain parameters, \% } \\
\hline $\mathrm{LV}_{\mathrm{LAX}}-\mathrm{GLS}$ & $-21.6(-23.2,-19.3)$ & $-18.4(-20.8,-15.5)$ & 0.0043 \\
\hline $\mathrm{LV}_{\mathrm{LAX}}-\mathrm{GCS}$ & $-26.1(-27.7,-22.9)$ & $-22.2(-25.5,-19.2)$ & 0.0039 \\
\hline $\mathrm{LV}_{\mathrm{LAX}}-\mathrm{GRS}$ & $107.0(82.5-126.1)$ & $84.5(68.4-104.0)$ & 0.0198 \\
\hline $\mathrm{LV}_{\mathrm{SAX}}-\mathrm{GCS}$ & $-23.4(-25.2,-21.8)$ & $-21.3(-24.0,-17.4)$ & 0.0125 \\
\hline $\mathrm{LV}_{\mathrm{SAX}}-\mathrm{GRS}$ & $102.3(78.0-126.9)$ & $82.0(62.0-108.5)$ & 0.0292 \\
\hline $\mathrm{LA}_{2 \mathrm{CH}^{-}}-\mathrm{GLS}$ & $37.2(33.0-43.7)$ & $27.0(16.1-34.5)$ & 0.0002 \\
\hline $\mathrm{LA}_{4 \mathrm{CH}^{-}}-\mathrm{GLS}$ & $28.8(22.8-41.8)$ & $21.8(13.2-29.0)$ & 0.0051 \\
\hline RV-GLS & $-37.6(-44.8,-32.6)$ & $-38.0(-43.6,-33.9)$ & 0.6577 \\
\hline RA-GLS & $33.7(28.9-42.0)$ & $30.9(22.5-39.1)$ & 0.0739 \\
\hline
\end{tabular}

Statistical significance was defined as $p<0.05$. Significant results are highlighted in bold

Values are median [first (Q1) and third (Q3) quartiles] for continuous and $n$ (\% of total column number) for categorical data

$H C M$ indicates hypertrophic cardiomyopathy, $b p m$ beats per minute, $h s$ high-sensitive, $N T N$-terminal, $L V$ left ventricular, $R V$ right ventricular, $L A$ left atrial, $R A$ right atrial, $E F$ ejection fraction, $E D V i$ end-diastolic volume index, ESVi end-systolic volume index, $L V W T$ left ventricular wall thickness, $D D$ diastolic 
Table 2 (continued)

dysfunction, NYHA New York Heart Association, LGE late gadolinium enhancement, SCD-Score sudden cardiac death score, $L A X$ longitudinal axis, $S A X$ short axis, $G L S$ global longitudinal strain, $G C S$ global circumferential strain, $G R S$ global radial strain, $2 \mathrm{CH}$ two-chamber view, $4 \mathrm{CH}$ four-chamber view
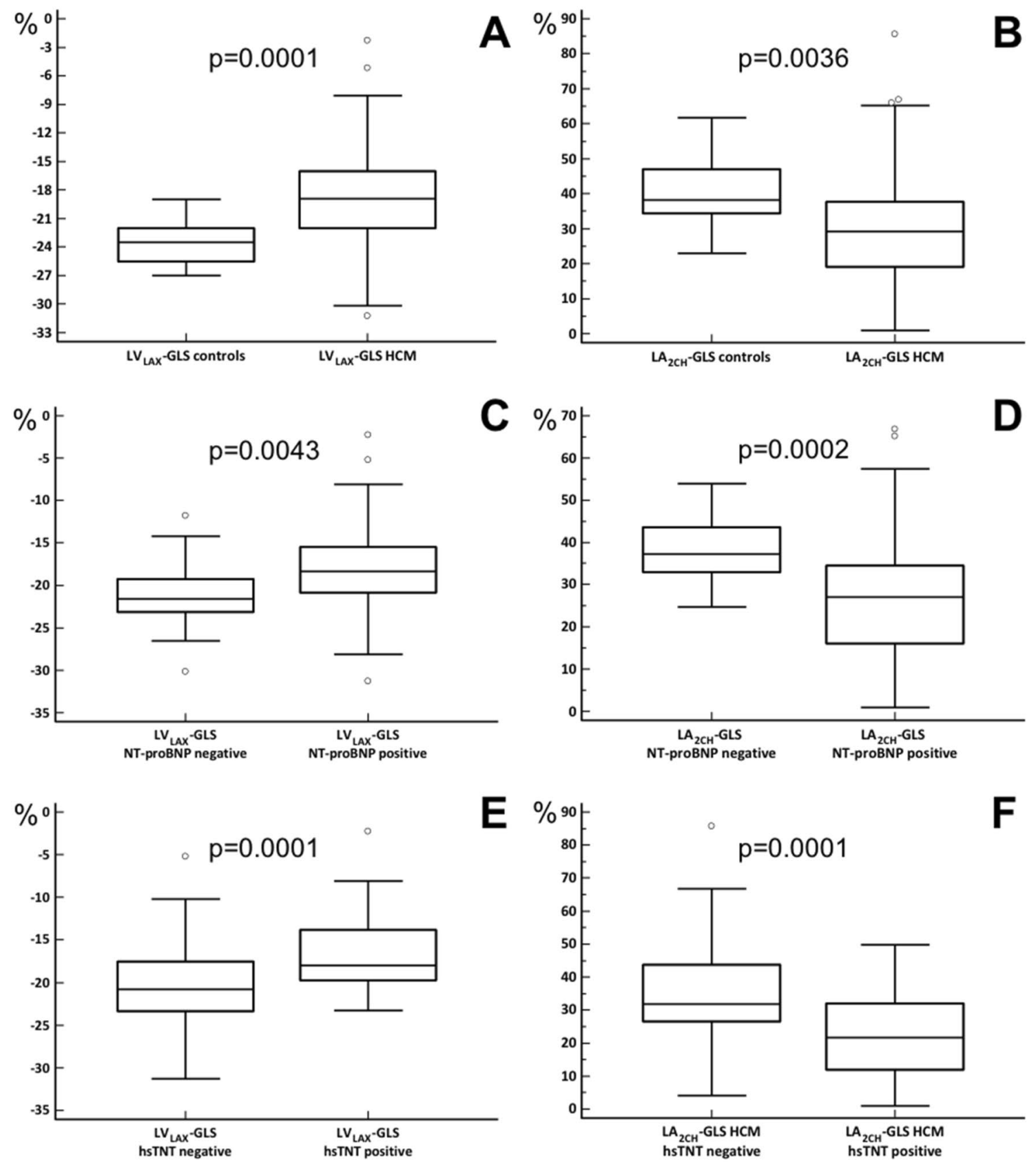

Fig. 1 Distribution of LV and LA strain. Box-Whisker plots of median $\mathrm{LV}_{\mathrm{LAX}}$-GLS and $\mathrm{LA}_{2 \mathrm{CH}^{-}}$GLS in controls and $\mathrm{HCM}(\mathbf{a}, \mathbf{b})$ and in HCM with negative $(<125 \mathrm{pg} / \mathrm{mL})$ NT-proBNP blood levels com-

further investigation. Taken together, our findings suggest that CMR-FT identifies LV and RV functional changes in HCM independent from conventional parameters. CMR-FT contributes to a better understanding of pathophysiology and could potentially improve risk stratification in HCM.

pared to HCM with elevated (positive) NT-proBNP levels (c, d) as well as in HCM with negative hsTnT $(<14 \mathrm{pg} / \mathrm{mL})$ or elevated (positive) hsTnT (e, f)

\section{Atrial strain in HCM}

We found that $\mathrm{LA}_{2 \mathrm{CH}^{-}} \mathrm{GLS}$ and $\mathrm{LA}_{4 \mathrm{CH}^{-}}-\mathrm{GLS}$ were significantly lower in patients with HCM compared to controls. Quantification of LA Strain is a sensitive marker of LV diastolic dysfunction independent of LVEF [26]. In our 

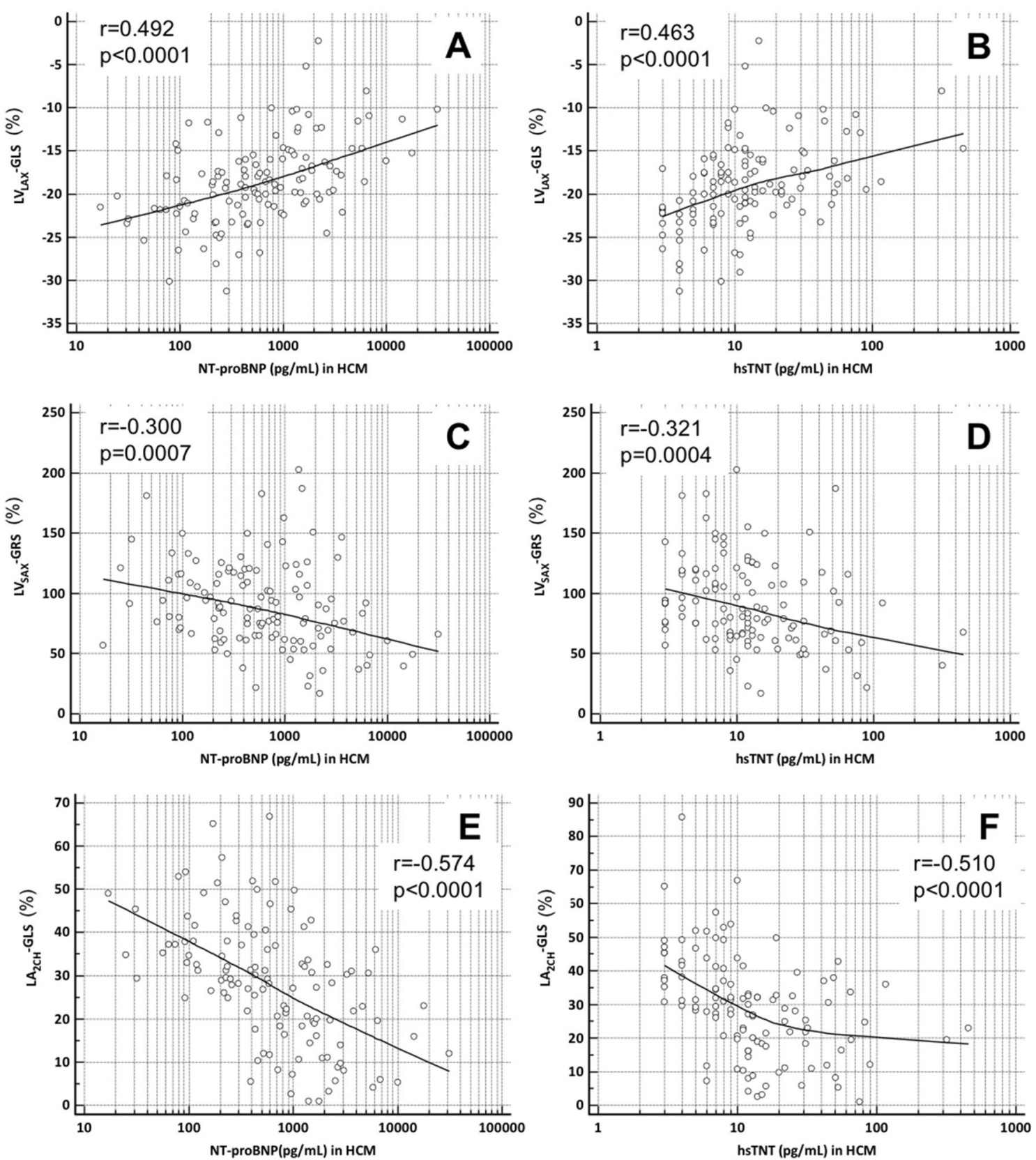

Fig. 2 Correlation of $\mathrm{LV}_{\mathrm{LAX}} \mathrm{GLS}, \mathrm{LV}_{\mathrm{SAX}} \mathrm{GRS}$ and $\mathrm{LA}_{2 \mathrm{CH}} \mathrm{GLS}$ with NT-proBNP and hsTnT. Scatterplots of $\mathrm{LV}_{\mathrm{LAX}} \mathrm{GLS}, \mathrm{LV}_{\mathrm{SAX}} \mathrm{GRS}$, $\mathrm{LA}_{2 \mathrm{CH}} \mathrm{GLS}$ and circulation biomarkers such as NT-proBNP and highsensitivity cardiac troponin T (hsTnT). Spearman's correlations were a LV LAX $_{\text {GLS }}$ and NT-proBNP: $r=0.492, p<0.0001$; b $\mathrm{LV}_{\mathrm{LAX}} \mathrm{GLS}$

study, the majority of HCM patients (92\%) had advanced diastolic dysfunction (Table 2). Interestingly, Habibi et al. demonstrated that deteriorations in LA-GLS precede the development of HF [27]. It is also known, that LA-GLS is predictive of worsening $\mathrm{HF}$ in patients with HCM [28]. Taking these results into account, $\mathrm{LA}_{2 \mathrm{CH}^{-}} \mathrm{GLS}$ and $\mathrm{LA}_{4 \mathrm{CH}^{-}}$GLS may not only reflect diastolic LV dysfunction in HCM but could also serve as an early predictor of HF and hsTnT: $r=0.463, p<0.0001$; LV $_{\mathrm{SAX}} \mathrm{GRS}$ and NT-proBNP: $r=-0.300, p=0.0007 ; \mathbf{d} \mathrm{LV}_{\mathrm{SAX}} \mathrm{GRS}$ and hsTnT: $r=-0.321$, $p=0.0004$; e LA ${ }_{2 \mathrm{CH}} \mathrm{GLS}$ and NT-proBNP: $r=-0.574, p<0.0001$; f LA $\mathrm{LAH}_{2 \mathrm{H}} \mathrm{GLS}$ and hsTnT: $r=-0.510, p<0.0001$. Note: logarithmic transformation of $x$-axis

and atrial fibrillation risk in HCM patients. Longitudinal studies are necessary in this context. However, we did not find significant difference in RA-GLS between HCM and controls (Table 1). Median RA-GLS was similar to previously described reference values [29]. We assume that the role of RA function in the pathophysiology of HCM is marginal. 
Table 3 Clinical and CMR characteristics of HCM patients with and without elevated hsTnT

\begin{tabular}{|c|c|c|c|}
\hline Parameter, unit & $\begin{array}{l}\text { HCM with hsTnT }<14 \mathrm{pg} / \mathrm{mL} \\
(n=75)\end{array}$ & $\begin{array}{l}\text { HCM with hsTnT } \geq 14 \mathrm{pg} / \mathrm{mL} \\
(n=44)\end{array}$ & $p$ value \\
\hline Age, years & $53(41-63)$ & $59(53-67)$ & 0.0471 \\
\hline Male, $n,(\%)$ & $48 / 75(64 \%)$ & $26 / 44(59 \%)$ & 0.6958 \\
\hline Heart rate, bpm & $65(60-72)$ & $66(58-76)$ & 0.7557 \\
\hline Height, m & $1.74(1.66-1.82)$ & $1.75(1.65-1.83)$ & 0.7809 \\
\hline Weight, $\mathrm{kg}$ & $82(74-92)$ & $84(75-92)$ & 0.9079 \\
\hline Creatinine, $\mathrm{mg} / \mathrm{dL}$ & $0.9(0.75-1.09)$ & $1.0(0.80-1.16)$ & 0.1255 \\
\hline $\mathrm{GFR}, \mathrm{mL} / \mathrm{min}$ & $85(68-101)$ & $74(62-99)$ & 0.0375 \\
\hline Creatine kinase, U/L & $113(79-169)$ & $115(84-174)$ & 0.6362 \\
\hline NT-proBNP, pg/mL & $421(136-961)$ & $1228(459-2451)$ & $<0.0001$ \\
\hline \multicolumn{4}{|l|}{ Volumes and functions } \\
\hline LVEF, \% & $66(58-72)$ & $62(57-66)$ & 0.0138 \\
\hline LVEDVi, $\mathrm{mL} / \mathrm{m}^{2}$ & $85(76-96)$ & $92(81-106)$ & 0.0445 \\
\hline LVESVi, $\mathrm{mL} / \mathrm{m}^{2}$ & $28(24-35)$ & $36(28-46)$ & 0.0016 \\
\hline LV Mass Index, $\mathrm{g} / \mathrm{m}^{2}$ & $71(55-86)$ & $83(70-109)$ & 0.0013 \\
\hline Maximal LVWT, mm & $17.3(15.3-20.7)$ & $19.1(15.9-21.4)$ & 0.1831 \\
\hline RVEF, \% & $64(58-68)$ & $64(53-70)$ & 0.8017 \\
\hline $\mathrm{RVEDVi}, \mathrm{mL} / \mathrm{m}^{2}$ & $72(64-87)$ & $74(65-91)$ & 0.5244 \\
\hline RVESVi, $\mathrm{mL} / \mathrm{m}^{2}$ & $26(23-34)$ & $29(22-37)$ & 0.4360 \\
\hline $\mathrm{LAEDVi}, \mathrm{mL} / \mathrm{m}^{2}$ & $27(20-43)$ & $45(28-65)$ & 0.0011 \\
\hline LAESVi, $\mathrm{mL} / \mathrm{m}^{2}$ & $50(42-63)$ & $64(48-90)$ & 0.0115 \\
\hline $\mathrm{RAEDVi}, \mathrm{mL} / \mathrm{m}^{2}$ & $20(16-27)$ & $24(18-39)$ & 0.0582 \\
\hline $\mathrm{RAESVi}, \mathrm{mL} / \mathrm{m}^{2}$ & $38(28-47)$ & $36(26-58)$ & 0.5691 \\
\hline Normal diastolic function, $n,(\%)$ & $0 / 57(0)$ & $1 / 32(3.1)$ & 0.3596 \\
\hline $\mathrm{DD} \mathrm{I}, n,(\%)$ & $2 / 57(3.5)$ & $5 / 32(15.6)$ & 0.0930 \\
\hline $\mathrm{DD} \mathrm{II}, n,(\%)$ & $46 / 57(80.7)$ & $21 / 32(65.6)$ & 0.1308 \\
\hline $\mathrm{DD} \mathrm{III}^{\circ}, n,(\%)$ & $9 / 57(15.8)$ & $5 / 32(15.6)$ & 1.0000 \\
\hline Diastol dysfunction $\geq \mathrm{II}^{\circ}, n,(\%)$ & $55 / 57(96.5)$ & $26 / 32(81.3)$ & 0.0233 \\
\hline NYHA I, $n,(\%)$ & 26/73 (35.6) & $13 / 40(32.5)$ & 0.8371 \\
\hline NYHA II, $n,(\%)$ & $33 / 73(45.2)$ & $19 / 40(47.5)$ & 0.8456 \\
\hline NYHA III, $n,(\%)$ & $14 / 73(19.2)$ & $8 / 40(20)$ & 0.4335 \\
\hline NYHA IV, $n,(\%)$ & 0/73 (0) & $0 / 40(0)$ & 1.0000 \\
\hline LGE presence, n, (\%) & $51 / 74(69)$ & $35 / 44(80)$ & 0.2847 \\
\hline $\begin{array}{l}\text { LGE extent, } n \text { (amount of LGE segments } \\
\text { in } \% \text { ) }\end{array}$ & $\begin{array}{l}129 / 1258 \\
(10)\end{array}$ & $\begin{array}{l}118 / 748 \\
(16)\end{array}$ & 0.0003 \\
\hline Atrial fibrillation, $n,(\%)$ & $2 / 75(2.7)$ & $5 / 44(11.4)$ & 0.1117 \\
\hline HCM-SCD-Score, $\%$ & $2.9(2.1-5.1)$ & $3.3(2.2-5.3)$ & 0.3997 \\
\hline LVOT obstruction, $n,(\%)$ & $26 / 75(34.7)$ & $15 / 44(34.1)$ & 1.0000 \\
\hline \multicolumn{4}{|l|}{ Strain parameters, $\%$} \\
\hline $\mathrm{LV}_{\mathrm{LAX}}-\mathrm{GLS}$ & $-20.8(-23.4,-19.0)$ & $-18.0(-19.7,-13.8)$ & 0.0001 \\
\hline $\mathrm{LV}_{\mathrm{LAX}}-\mathrm{GCS}$ & $-24.7(-26.9,-22.0)$ & $-20.5(-24.3,-17.1)$ & 0.0001 \\
\hline $\mathrm{LV}_{\text {LAX }}-\mathrm{GRS}$ & $91.2(76.4-115.1)$ & $79.8(58.9-96.4)$ & 0.0230 \\
\hline $\mathrm{LV}_{\mathrm{SAX}}-\mathrm{GCS}$ & $-22.2(-25.5,-19.7)$ & $-21.0(-23.8,-16.4)$ & 0.0217 \\
\hline $\mathrm{LV}_{\mathrm{SAX}}-\mathrm{GRS}$ & $93.5(70.0-119.7)$ & $72.0(53.3-97.0)$ & 0.0020 \\
\hline $\mathrm{LA}_{2 \mathrm{CH}^{-}} \mathrm{GLS}$ & $31.8(26.5-43.7)$ & $21.8(11.9-32.0)$ & 0.0001 \\
\hline $\mathrm{LA}_{4 \mathrm{CH}^{-}}-\mathrm{GLS}$ & $25.3(18.4-31.6)$ & $16.4(12.6-26.1)$ & 0.0021 \\
\hline RV-GLS & $-38.3(-44.1,-33.7)$ & $-38.3(-43.9,-34.2)$ & 0.9015 \\
\hline RA-GLS & $33.9(26.4-43.1)$ & $26.5(22.0-32.1)$ & 0.0011 \\
\hline
\end{tabular}

Statistical significance was defined as $p<0.05$. Significant results are highlighted in bold

Values are median [first (Q1) and third (Q3) quartiles] for continuous and $n$ (\% of total column number) for categorical data

$H C M$ indicates hypertrophic cardiomyopathy, $b p m$ beats per minute, $h s$ high-sensitivity, $N T N$-terminal, $L V$ left ventricular, $R V$ right ventricular, $L A$ left atrial, $R A$ right atrial, $E F$ ejection fraction, $E D V i$ enddiastolic volume index, $E S V i$ end-systolic volume index, $L V W T$ left ventricular wall thickness, $D D$ diastolic dysfunction, NYHA New York Heart Association, $L G E$ late gadolinium enhancement, SCD-Score sudden 
Table 3 (continued)

Fig. 3 Deformation mechanics in control and in HCM. a Diastolic phase in control; LV is relaxing homogeneously. b Systolic phase in control; LV is contracting with a shortening in the longitudinal axis. c Diastolic phase in HCM; impaired relaxation of the hypertrophied LV septum. d Systolic phase in HCM; impaired contraction of the hypertrophied LV septum, compensatory hypercontractile motion of non-hypertrophied LV segments, impaired longitudinal shortening. Note, length of arrows displays relative extent of deformation cardiac death score, $L A X$ longitudinal axis, $S A X$ short axis, GLS global longitudinal strain, GCS global circumferential strain, $G R S$ global radial strain, $2 \mathrm{CH}$ two-chamber view, $4 \mathrm{CH}$ four-chamber view

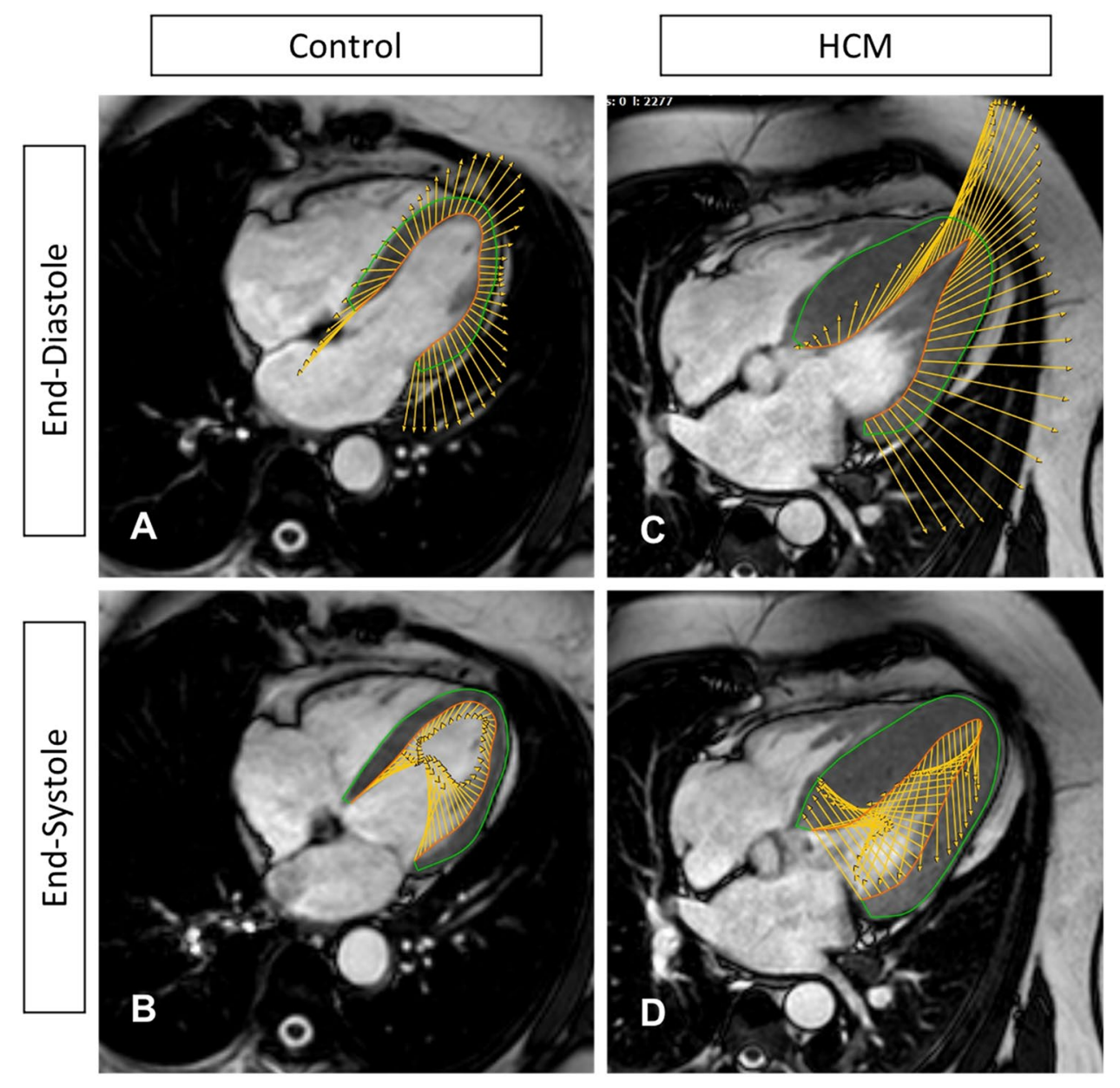

\section{Myocardial strain and the association with cardiac biomarkers}

NT-proBNP is a major cardiac biomarker, that is not only used to diagnose HF [10] but also as a powerful prognostic factor in different cardiomyopathies [30]. Cardiac hsTnT is a crucial marker of myocardial injury and is linked to poor outcome in ischemic as well as non-ischemic cardiomyopathies [31, 32]. We found that all global CMR-FT parameters of the LV, but not conventional LVEF and diastolic function, were significantly impaired in HCM patients with elevated biomarker concentrations (Tables 2, 3). Furthermore, we found a significant correlation of biomarker levels with CMR-FT (Fig. 2). These findings are in line with recent STI and CMR-FT studies, in which reduced GLS, GCS and GRS in HCM were independent predictors of poor cardiac outcome, particularly HF [6, 33]. Furthermore, in a recent CMR-FT study Tanacli et al. demonstrated that LV GLS and GCS drop with the severity of HF [34]. Our findings indicate that LV CMR-FT strain reveals occult systolic dysfunction in HCM. Furthermore, diastolic dysfunction is very common in HCM (Table 2) and LA-GLS seems to be a suitable strain parameter to evaluate diastolic dysfunction [26]. In conclusion, alterations in myocardial strain and elevated biomarkers seem to depict patients with more severe disease, independent from conventional assessment such as LVEF, NYHA classification, LVOT obstruction or the SCD risk score. Future studies are necessary to assess the potential prognostic implications of these findings.

\section{Limitations}

This study is a retrospective study with its inherent limitations, such as missing values of cardiac biomarkers in some patients. Furthermore, this study did not include longitudinal, follow-up data and is, therefore, not designed to address a potential incremental value of CMR-FT strain over conventional imaging. Future longitudinal studies are needed to assess a potential incremental prognostic value of CMRFT over conventional imaging such as the assessment of 

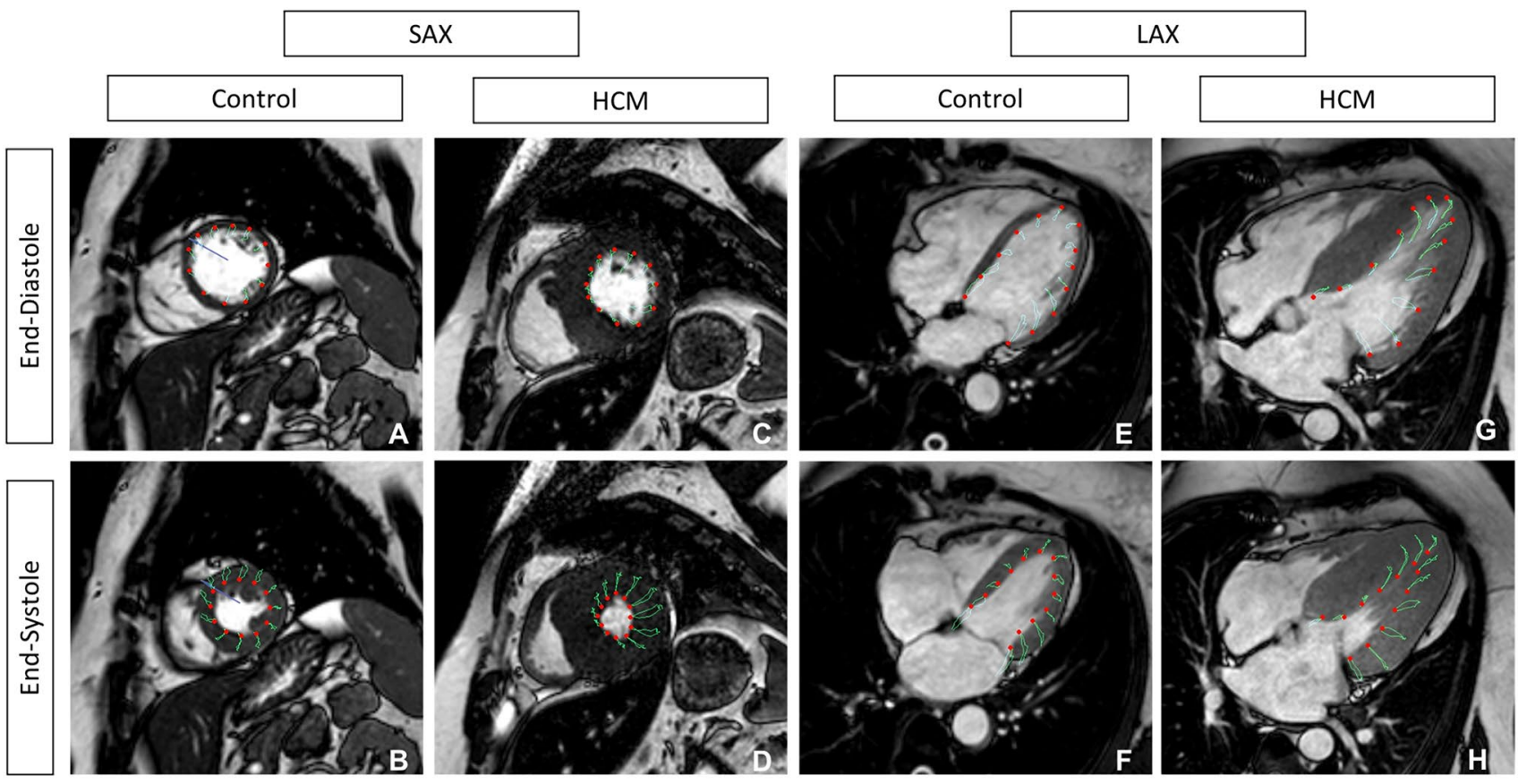

Fig. 4 Typical LV-strain findings in HCM in comparison to a control. a-d Short-axis (SAX) left midventricular (LV) cine images of a control $(\mathbf{a}, \mathbf{b})$ and a HCM patient in end-diastole and end-systole $(\mathbf{c}, \mathbf{d})$. Note, the typical asymmetric septal LV hypertrophy $(\mathbf{c}, \mathbf{d})$. e-h Long-

diastolic dysfunction by echocardiography. To date, CMRFT strain is often used in addition to conventional imaging parameters since there is currently no clinical application with immediate individual benefit for patients. In particular, there is a substantial overlap in some strain parameters between HCM patients and controls. However, strain reflects the myocardial deformation process much more accurately compared to conventional imaging and may therefore contribute to a better understanding of subclinical pathophysiological myocardial alterations. In addition, T1-mapping/ ECV imaging was not available before 2011 in this study population. Therefore, we were not able to systematically address potential associations between strain parameters and quantitative tissue characterization in this study. Long-axisbased GLS is currently perceived as the most robust and reproducible CMR-FT parameter, but the reproducibility of other strain parameters can differ significantly $[12,35]$. We found that $\mathrm{LV}_{\mathrm{LAX}}-\mathrm{GRS}$ and $\mathrm{LV}_{\mathrm{SAX}}-\mathrm{GCS}$ were affected by a poor and moderate inter-observer agreement in our study population, respectively. However, there were good to excellent inter-observer agreements for all other CMR-FT-derived measurements in this study population, which supports the reliability of the major findings of this work. axis (LAX) LV strain of the same control (e, f) and the same HCM patient $(\mathbf{g}, \mathbf{h})$. Note, red dots follow green lines and display myocardial deformation within one heart cycle. Septal deformation is lower than i.e., lateral deformation, shown by shorter green lines

\section{Conclusion}

CMR-FT reveals LV and LA dysfunction in HCM patients despite normal LVEF. The association between impaired LV strain and elevated NT-proBNP and hsTnT levels indicates a link between unapparent functional abnormalities and disease severity in HCM.

Acknowledgements We cordially thank Miriam Wendner and the team of the cardiological outpatient department for their contribution to clinical data collection.

Funding Open Access funding enabled and organized by Projekt DEAL.

\section{Declarations}

\section{Conflict of interest None.}

Open Access This article is licensed under a Creative Commons Attribution 4.0 International License, which permits use, sharing, adaptation, distribution and reproduction in any medium or format, as long as you give appropriate credit to the original author(s) and the source, provide a link to the Creative Commons licence, and indicate if changes were made. The images or other third party material in this article are included in the article's Creative Commons licence, unless indicated otherwise in a credit line to the material. If material is not included in the article's Creative Commons licence and your intended use is not permitted by statutory regulation or exceeds the permitted use, you will 
need to obtain permission directly from the copyright holder. To view a copy of this licence, visit http://creativecommons.org/licenses/by/4.0/.

\section{References}

1. Wigle ED, Silver MD (1978) Myocardial fiber disarray and ventricular septal hypertrophy in asymmetrical hypertrophy of the heart. Circulation 58(3):398-402

2. Elliott PM, Anastasakis A, Borger MA, Borggrefe M, Cecchi F et al (2014) 2014 ESC Guidelines on diagnosis and management of hypertrophic cardiomyopathy. Eur Heart J 35(39):2733-79

3. Kalam K, Otahal P, Marwick TH (2014) Prognostic implications of global LV dysfunction: a systematic review and metaanalysis of global longitudinal strain and ejection fraction. Heart 100(21):1673-1680

4. Saito M, Okayama H, Yoshii T, Higashi H, Morioka H, Hiasa $\mathrm{G}$ et al (2012) Clinical significance of global two-dimensional strain as a surrogate parameter of myocardial fibrosis and cardiac events in patients with hypertrophic cardiomyopathy. Eur Heart J Cardiovasc Imaging 13(7):617-623

5. Pedrizzetti G, Claus P, Kilner PJ, Nagel E (2016) Principles of cardiovascular magnetic resonance feature tracking and echocardiographic speckle tracking for informed clinical use. J Cardiovasc Magn Reson 18(1):1-12

6. Hinojar R, Fernández-Golfín C, González-Gómez A, Rincón LM, Plaza-Martin M, Casas E et al (2017) Prognostic implications of global myocardial mechanics in hypertrophic cardiomyopathy by cardiovascular magnetic resonance feature tracking. Relations to left ventricular hypertrophy and fibrosis. Int J Cardiol. 249:467-72

7. Radunski UK, Fuger U, Bohnen S, Lund GK, Stehning C, Zeller T et al (2017) Asymptomatic cocaine abuse: myocardial tissue characterization using cardiac biomarkers and cardiovascular magnetic resonance imaging. Circ J 81(5):701-708

8. Nagueh SF, Smiseth OA, Appleton CP, Byrd BF, Dokainish H, Edvardsen $T$ et al (2016) Recommendations for the evaluation of left ventricular diastolic function by echocardiography: an update from the American Society of Echocardiography and the European Association of Cardiovascular Imaging. J Am Soc Echocardiogr 29(4):277-314

9. Body R, Burrows G, Carley S, Cullen L, Than M, Jaffe AS et al (2015) High-sensitivity cardiac Troponin T concentrations below the limit of detection to exclude acute myocardial infarction : a prospective evaluation. Clin Chem 989:983-989

10. Ponikowski P, Voors AA, Anker SD, Bueno H, Cleland JGF, Coats AJS et al (2016) 2016 ESC Guidelines for the diagnosis and treatment of acute and chronic heart failure. Eur Heart J 37(27):2129-2200m

11. Schulz-Menger J, Bluemke DA, Bremerich J, Flamm SD, Fogel MA, Friedrich MG et al (2020) Standardized image interpretation and post-processing in cardiovascular magnetic resonance-2020 update: Society for Cardiovascular Magnetic Resonance (SCMR): Board of Trustees Task Force on Standardized Post-Processing. J Cardiovasc Magn Reson 22(1):1-22

12. Barreiro-Pérez M, Curione D, Symons R, Claus P, Voigt JU, Bogaert J (2018) Left ventricular global myocardial strain assessment comparing the reproducibility of four commercially available CMR-feature tracking algorithms. Eur Radiol 28(12):5137-5147

13. Claus P, Omar AMS, Pedrizzetti G, Sengupta PP, Nagel E (2015) Tissue tracking technology for assessing cardiac mechanics principles, normal values, and clinical applications. JACC Cardiovasc Imaging 8(12):1444-1460
14. Scatteia A, Baritussio A, Bucciarelli-Ducci C (2017) Strain imaging using cardiac magnetic resonance. Heart Fail Rev 22(4):465-476

15. MacEira AM, Cosín-Sales J, Roughton M, Prasad SK, Pennell DJ (2013) Reference right atrial dimensions and volume estimation by steady state free precession cardiovascular magnetic resonance. J Cardiovasc Magn Reson 15(1):1-10

16. Kowallick JT, Kutty S, Edelmann F, Chiribiri A, Villa A, Steinmetz M et al (2014) Quantification of left atrial strain and strain rate using cardiovascular magnetic resonance myocardial feature tracking: a feasibility study. J Cardiovasc Magn Reson 16(1):1-9

17. Selvadurai BSN, Puntmann VO, Bluemke DA, Ferrari VA, Friedrich MG, Kramer CM et al (2018) Definition of left ventricular segments for cardiac magnetic resonance imaging. JACC Cardiovasc Imaging 11(6):926-928

18. Koo TK, Li MY (2016) A guideline of selecting and reporting intraclass correlation coefficients for reliability research. J Chiropr Med 15(2):155-163

19. Wehner GJ, Jing L, Haggerty CM, Suever JD, Leader JB, Hartzel DN et al (2020) Routinely reported ejection fraction and mortality in clinical practice: Where does the nadir of risk lie? Eur Heart J 41(12):1249-1257

20. Pryds K, Larsen AH, Hansen MS, Grøndal AYK, Tougaard RS, Hansson NH et al (2019) Myocardial strain assessed by feature tracking cardiac magnetic resonance in patients with a variety of cardiovascular diseases - A comparison with echocardiography. Sci Rep 9(1):11296

21. Romano S, Judd RM, Kim RJ, Kim HW, Klem I, Heitner JF et al (2018) Feature-tracking global longitudinal strain predicts death in a multicenter population of patients with ischemic and nonischemic dilated cardiomyopathy incremental to ejection fraction and late gadolinium enhancement. JACC Cardiovasc Imaging 11(10):1419-1429

22. Neisius U, Myerson L, Fahmy AS, Nakamori S, El-Rewaidy H, Joshi $G$ et al (2019) Cardiovascular magnetic resonance feature tracking strain analysis for discrimination between hypertensive heart disease and hypertrophic cardiomyopathy. PLoS One 14(8):1-18

23. Almaas VM, Haugaa KH, Strøm EH, Scott H, Smith H, Dahl $\mathrm{CP}$ et al (2014) Noninvasive assessment of myocardial fibrosis in patients with obstructive hypertrophic cardiomyopathy. Heart 100(8):631-638

24. Guendouz S, Rappeneau S, Nahum J, Dubois-Randé JL, Gueret P, Monin JL et al (2012) Prognostic significance and normal values of $2 \mathrm{D}$ strain to assess right ventricular systolic function in chronic heart failure. Circ J 76(1):127-136

25. Yang L, Zhang L, Cao S, Gao C, Xu H, Song T et al (2020) Advanced myocardial characterization in hypertrophic cardiomyopathy: feasibility of CMR-based feature tracking strain analysis in a case-control study. Eur Radiol 30(11):6118-6128

26. Frydas A, Morris DA, Belyavskiy E, Radhakrishnan AK, Kropf M, Tadic M et al (2020) Left atrial strain as sensitive marker of left ventricular diastolic dysfunction in heart failure. ESC Hear Fail 7(4):1956-1965

27. Habibi M, Chahal H, Opdahl A, Gjesdal O, Helle-Valle TM, Heckbert SR et al (2014) Association of CMR-measured LA function with heart failure development. JACC Cardiovasc Imaging 7(6):570-579

28. Kobayashi Y, Moneghetti KJ, Bouajila S, Stolfo D, Finocchiaro G, Kuznetsova T et al (2019) Time based versus strain based myocardial performance indices in hypertrophic cardiomyopathy, themerging role of left atrial strain. Eur Heart J Cardiovasc Imaging 20(3):334-342

29. Truong VT, Palmer C, Young M, Wolking S, Ngo TNM, Sheets B et al (2020) Right atrial deformation using cardiovascular magnetic resonance myocardial feature tracking compared with 
two-dimensional speckle tracking echocardiography in healthy volunteers. Sci Rep 10(1):1-7

30. Kang SH, Park JJ, Choi DJ, Yoon CH, Oh IY, Kang SM et al (2015) Prognostic value of NT-proBNP in heart failure with preserved versus reduced EF. Heart 101(23):1881-1888

31. Kubo T, Kitaoka H, Yamanaka S, Hirota T (2013) Significance of high-sensitivity cardiac Troponin $\mathrm{T}$ in hypertrophic cardiomyopathy. J Am Coll Cardiol 62(14):1252-1259

32. Welsh P, Preiss D, Hayward C, Shah ASV, McAllister D, Briggs A et al (2019) Cardiac Troponin $\mathrm{T}$ and Troponin I in the general population: comparing and contrasting their genetic determinants and associations with outcomes. Circulation 139(24):2754-2764

33. Reant P, Mirabel M, Lloyd G, Peyrou J, Lopez Ayala JM, Dickie $S$ et al (2016) Global longitudinal strain is associated with heart failure outcomes in hypertrophic cardiomyopathy. Heart 102(10):741-747

34. Tanacli R, Hashemi D, Lapinskas T, Edelmann F, Gebker R, Pedrizzetti G et al (2019) Range variability in CMR feature tracking multilayer strain across different stages of heart failure. Sci Rep 9(1):16478

35. Lim C, Blaszczyk E, Riazy L, Wiesemann S, Schüler J, von Knobelsdorff-Brenkenhoff F et al (2020) Quantification of myocardial strain assessed by cardiovascular magnetic resonance feature tracking in healthy subjects-influence of segmentation and analysis software. Eur Radiol. 